\title{
The Role of Radiographic Phase-Contrast Imaging in the Development of Intracochlear Electrode Arrays
}

\author{
$* \dagger$ Jin Xu, $\ddagger$ Andrew W. Stevenson, $\ddagger$ Dachao Gao, *Michael Tykocinski, \\ $\dagger$ David Lawrence, $\ddagger$ Stephen W. Wilkins, $* \dagger$ Graeme M. Clark, *Elaine Saunders, \\ and *Robert S. Cowan \\ * Co-operative Research Center for Cochlear Implant and Hearing Aid Innovation, University of \\ Melbourne, tThe Bionic Ear Institute, and $¥ C S I R O$ Manufacturing Science and Technology, Clayton, \\ Victoria, Australia
}

Objective: This study describes the application of a new radiographic imaging modality, phase-contrast radiography, to in vitro human temporal bone imaging and investigates its use in the development of new electrode arrays for cochlear implants. Background: The development of perimodiolar electrode arrays for cochlear implants requires detailed information from postoperative radiologic assessment on the position of the array in relation to the cochlear structures. Current standard radiographic techniques provide only limited details.

Materials and Methods: Nucleus standard electrode arrays and perimodiolar Contour electrode arrays were implanted into the scala tympani of 11 human temporal bones. Both conventional and phase-contrast radiographs were taken of each temporal bone for comparative purposes.

Results: Phase-contrast imaging provides better visualization of anatomic details of the inner ear and of the structure of the intracochlear electrode array, and better definition of electrode location in relation to cochlear walls.

Conclusion: Phase-contrast radiography offers significant improvement over conventional radiography in images of in vitro human temporal bones. It seems to be a valuable tool in the development of intracochlear electrode arrays and cochlear implant research. However, this new radiographic technique still requires certain computational and physics challenges to be addressed before its clinical use can be established. Key Words: Cochlear implant-Electrode position-ImagingImaging plate-Microfocus-Perimodiolar electrode-Phasecontrast radiography-Temporal bone.

Otol Neurotol 22:862-868, 2001.
In the development of advanced cochlear implants, improvements to the intracochlear electrode arrays are a key issue. To recognize a promising new design of intracochlear electrode array, it is imperative to know the exact anatomic position of the electrodes, the insertion depth, and any damage to cochlear structures that may occur. Currently, conventional radiography is the most common method for assessing the electrode position after cochlear implantation (1-3). However, conventional radiography cannot clearly demonstrate the exact anatomic position of the electrodes in relation to the fine bony structures of the cochlea and any possible insertioninduced damage to them. In the study described here, a new radiographic imaging modality, phase-contrast radiography (4), was applied to in vitro human temporal bone imaging.

This study was supported by the Cooperative Research Center for Cochlear Implant and Hearing Aid Innovation, which is funded by the Commonwealth Government of Australia.

Address correspondence and reprint requests to Dr. Jin Xu, Department of Otolaryngology, University of Melbourne. 32 Gisborne Street, East Melbourne, Victoria 3002, Australia.
Conventional radiography (Fig. 1A) relies on the principles of absorption contrast derived from density differences and variations in composition and thickness of the object. In comparison to conventional radiography, phase-contrast radiography $(4,5)$ uses a microfocus $x$-ray source to produce a coherent spherical wave. When the spherical wave passes through the object, the wave front becomes distorted by refraction. A relatively large object-image distance is used to allow further wave propagation and interference effects to occur, resulting in observable changes in intensity (phase contrast) in the image plane (Fig. IB). The imaging geometry used also provides an inherent magnification and improved signalto-noise ratio. This projection geometry also enables recording media such as imaging plates to be used, with their inherent advantages such as high sensitivity and large dynamic range, without the severe limitation imposed by their resolution (which is inferior to that of film, for example).

Studies by Wilkins et al. (4) and Gao et al. (5) demonstrated that phase-contrast imaging is able to improve the visibility of weakly absorbing features in small bio- 
A

\section{Conventional Radiography}

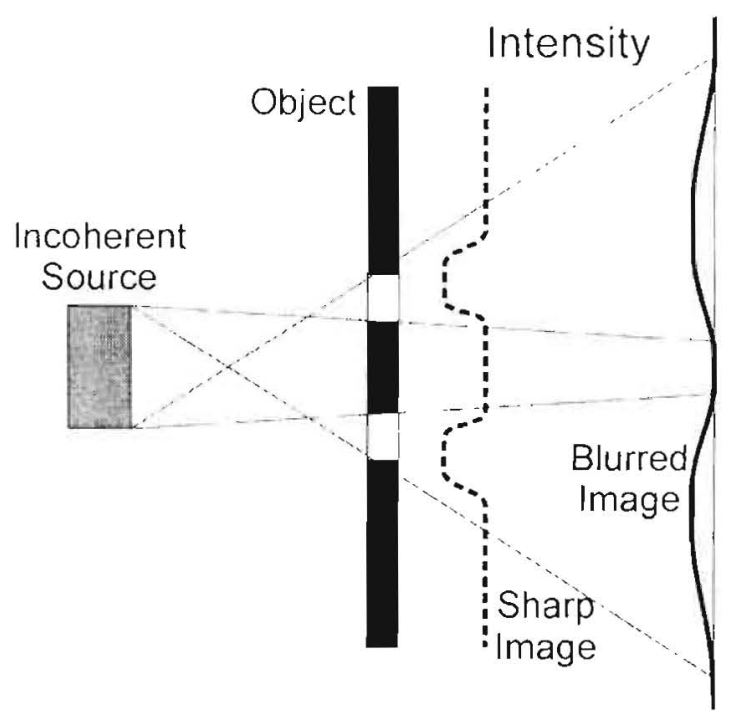

B Phase-Contrast Radiography

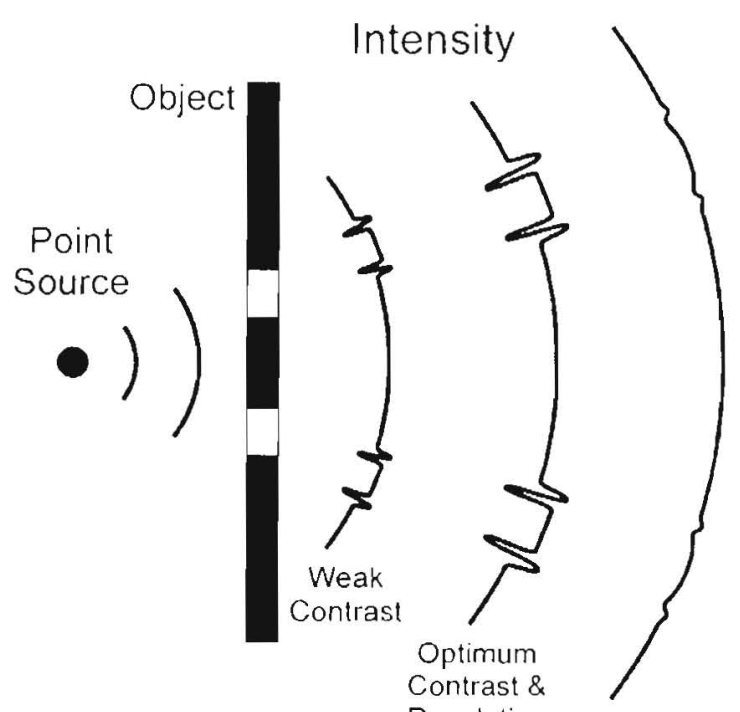

FIG. 1. A. Geometric optics representation of imaging of an absorptive object with an incoherent source. The resulting image is blurred when the object-image separation is large. B. Wave optics representation of imaging using a coherent source, demonstrating observable intensity variation caused by interference resulting from phase distortion of the wave front.

logic objects, for example, using goldfish, dragonfly, porcine liver, chicken knee, and human linger bone samples. In 1999, a preliminary study by Xu et al. (6) was conducted to investigate the possibility of using phase-contrast radiography for the imaging of human temporal bone: the bone with the most intricate anatomy and the highest bone density in the human body. The result was very encouraging. The study described here investigated the improvements that phase-contrast radiography can deliver for in vitro human temporal bone imaging and its potential role in the development of intracochlear electrode arrays for cochlear implants.

\section{MATERIAIS AND METHODS}

\section{Intracochlear electrode arrays}

The Nucleus standard electrode array (Fig. 2A) and the newly developed perimodiolar Contour clectrode array (Fig.
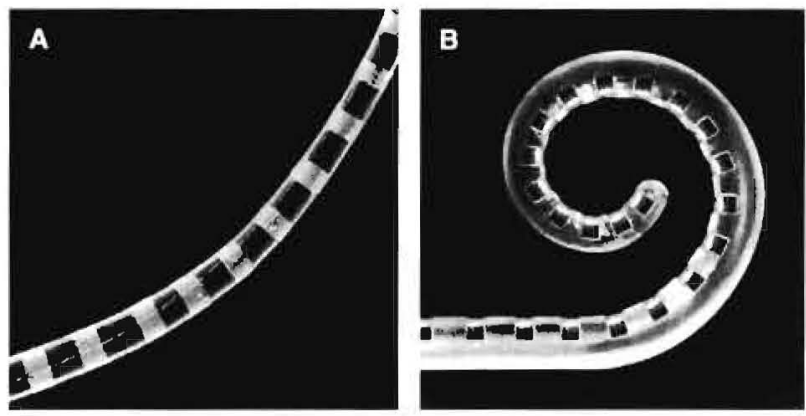

FIG. 2. Photomicrographs of Nucleus standard electrode array (A) and perimodiolar Contour electrode array (B)
2B) were used in this study. The straight standard electrode arraly comprises 22 full-band Platinum ( $\mathrm{Pt}$ ) electrodes ancl 10 nonaclive supporting bands, with the silicone carrier lapered from a tip dianeter of $0.4 \mathrm{~mm} 100.6 \mathrm{~mm}$ at the proximal end. The precurved Contour electrode array has 22 half-band $\mathrm{Pl}$ electrodes, oriented toward the modiolus, with the silicone carrier lapered from a tip diameter of $0.6100 .8 \mathrm{~mm}$ at the proximal end. The width of each electrode is $0.3 \mathrm{~mm}$ in both electrode designs. The diameter of the iridium-platinum wire connected io each electrode is $25 \mu \mathrm{m}$.

\section{Insertion of electrode arrays into temporal bones}

Lieven formalin-preserved human temporal hones were prepared as for cochlcar implant surgery. A standard Nucleus electrocle array was inserted into the cochlea of three temporal bones. The Nucleus Contour electrode array wals inserted into the cochlea of seven temporal bones. A cochlear surface preparation was performed under a microscope on six of these seven temporal bones alter insertion to assess electrode position and possible basilar membrane damage. No electrode array was inserted into the remaining temporal bone. A profite of those temporal bones is summarized in Table 1.

\section{Radiographic techniques}

Both a conventional radiograph and at phase-contrast radiograph were taken of each temporal bone for comparative pur-

TABI.E 1. Profite of 11 remporal bones

\begin{tabular}{|c|c|c|}
\hline Temporal bone & $\begin{array}{l}\text { Type of nucleus } \\
\text { electrode atray }\end{array}$ & $\begin{array}{c}\text { Cochleals surtace pupatration } \\
\text { (alfer insction) }\end{array}$ \\
\hline Temporial bone $1-\hat{i}$ & Slandiard & $N_{(1)}$ \\
\hline Tomporal bonc $f$ & Contour & $N_{0}$ \\
\hline Temporal bonc $5-10$ & Contour & YCu \\
\hline lemporial bone 1 | & No electrode & $\left.N_{1}\right)$ \\
\hline
\end{tabular}


TABIE 2. Technical profile of comventional radiography and phase-contrast radiography for typical cases used in the presem sums

\begin{tabular}{|c|c|c|}
\hline & Conventional radiography & $\begin{array}{l}\text { Phalse-contrast } \\
\text { rildiography }\end{array}$ \\
\hline Focial spot & $0.3 \times 1.0 \mathrm{~mm}$ & $4 \mu m$ \\
\hline Source-objecl dislance & $99 \mathrm{~cm}$ & $30 \mathrm{~cm}$ \\
\hline Object-image distance & $35 \mathrm{~cm}$ & $170 \mathrm{~cm}$ \\
\hline Exposure & $60 \mathrm{kV} .13 \mathrm{mAs}$ & $90 \mathrm{kV} .6 \mathrm{mAs}$ \\
\hline Recording medium & $\begin{array}{l}\text { Agfal Curix Otho Cassetle with fine } \\
\text { intensifying screen Kodak G/RA film }\end{array}$ & Fujifilm imaging plate BAS-SR (2025) \\
\hline Scanner & PowerLook2000 $(8.5 \mu \mathrm{m} /$ pixel $)$ & Fujifilm BAS-5000(25 $\mu \mathrm{m} /$ pixel $)$ \\
\hline
\end{tabular}

poses. An ultafine focus skull radiographic unit (Ultacranio: lsotopan Snc, S. Angelo Romano (Romal), ltaly) was used for laking conventional radiographs. The lïlms were scanned al $8.5-\mu \mathrm{m}$ pixel sizc with a PowerLook2(0)0 scanner. An indus- trial microliocus $x$-ray unil (Model FXE-225.20; Feinfocus Röntgen-Systeme GmbH. Girbsen. Germany) with stationary larget, and Fuji photostimulable phosphor imaging plates, were used for taking the phase-contrast radiographs. The imaging
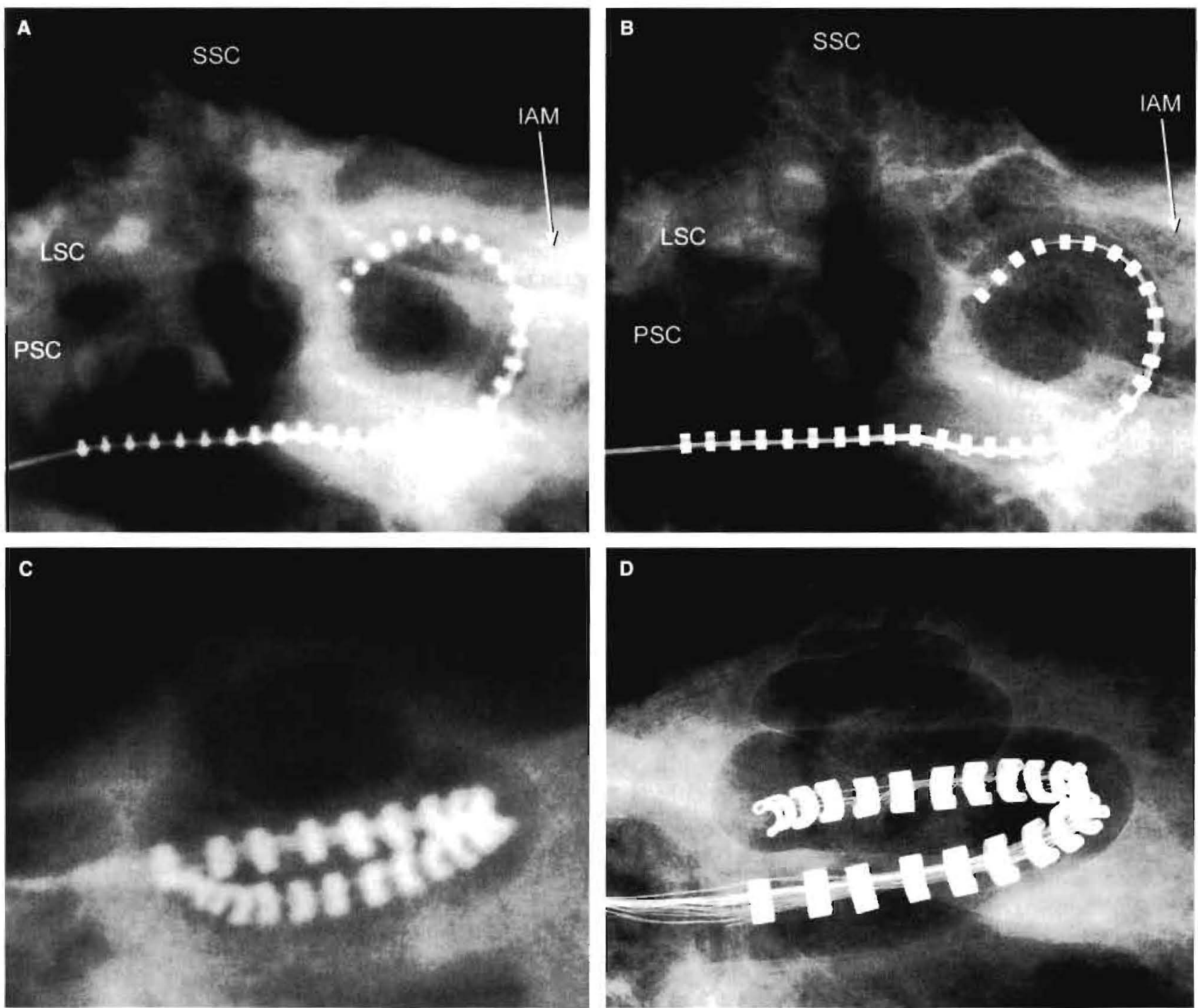

FIG. 3. Comparison of conventional radiograph $(\mathbf{A}, \mathbf{C})$ and phase-contrast radiograph $(\mathbf{B}, \mathbf{D})$ of the labyrinth part of temporal bone 1 implanted with an experimental Nucleus standard electrode array. (A,B, cochlear view) and the cochlear part of temporal bone 4 implanted with a prototype version of the Nucleus Contour electrode array (C,D, oblique view). SSC, superior semicircular canal; LSC, lateral semicircular canal; PSC, posterior semicircular canal; IAM, internal auditory meatus. 
plates were typically scanned at $25 \mu \mathrm{m}$ pixel size with a BAS5000 scanner. Technical profiles of both conventional radiography and phase-contrast radiography are detailed in Table 2 . The phase-contrast configuration described is the subject of an issued patent (7) and ongoing Patent Cooperation Treaty applications held by X-Ray Technologies Ply. Lid., Melbourne, Australia.

\section{RESULTS}

\section{Conventional versus phase-contrast radiographs}

Conventional and phase-contrast radiographs were obtained for each temporal bone. The significant portions of the images were enlarged to an appropriate size for comparison. Figure 3 shows the cochlear view (I) of the labyrinth part of temporal bone 1 implanted with a Nucleus standard electrode array and an oblique view of the cochlear part of temporal bone 4 implanted with an early prototype version of the Nucleus Contour electrode array.

It is apparent that the imaging contrast was much enhanced at the edge of canal-type structures, such as the semicircular canals, the cochlea, and the internal auditory meatus, by the use of phase-contrast radiography (Fig. $3 \mathrm{~B}, \mathrm{D}$ ) in comparison with conventional radiography (Fig. 3 A,C). A good example of such a sharp contrast, between the otic capsule and the air- or fluid-filled cochlear scalae, is evident in Figure 3D.

Figure 4 shows part of a Nucleus standard electrode array in the cochlea of temporal bone 1 (Fig. 4 C,D) and part of the Contour electrode array in the cochlea of temporal bone 4 (Fig. 4 A,B) at a higher magnification. The phase-contrast radiographs (Fig. 4 B,D) contain more details of the microstructures of the electrode array. Single iridium-platinum wires, which are only $25 \mu \mathrm{m}$ in diameter, can be clearly identified. By comparison, the platinum bands and wires are very blurry and indistinct in the conventional radiographs (Fig. 4 A,C).

It is obvious not only that the anatomic structures of the inner ear are shown in much greater detail by phasecontrast radiography, but also that the images of the electrode arrays are much more detailed. This advantage has not been observed with any of the other radiographic methods available to us previously.

\section{Position of the electrode array within the cochlea}

Figure 5 shows phase-contrast radiographs (cochlear view) of temporal bone 1 implanted with the Nucleus standard electrode array and temporal bone 6 implanted with the Nucleus Contour array. The enhanced imaging contrast at the edge of the cochlear wall using phasecontrast radiography enables better definition of the position of an electrode array in relation to the walls of the cochlea. The standard array in temporal bone I was Iocated along the outer wall of the cochlea (Fig. 5A), far from the neural elements. In temporal bone 6 , the Contour array (Fig. 5B) was inserted more deeply and positioned closer to the modiolus, compared with the standard array in temporal bone 1 (Fig. 5A).
A group of six temporal bones (temporal bones 5-10) was implanted with the Nucleus Contour electrode array. A surface preparation was performed on each temporal bone after electrode insertion. Figure 6 shows the phasecontrast radiographs of these six temporal bones (cochlear view orientation). The resultant images indicate that, in general, the Contour array lies close to the modiolus and achieves a greater insertion depth than does the standard Nucleus array. More details of two representative temporal bones are shown in Figures 7 and 8 .

The phase-contrast radiograph of temporal bone $6 \mathrm{im}$ planted with the Contour electrode array shows that the array is located close to the inner wall of the cochlea (Fig. 7A). Careful observation of the side view of the phase-contrast radiograph (Fig. 7B) shows that the osseous spiral lamina (OSL) is just visible, with the Contour array positioned under the OSL in the scala tympani. A cochlear surface preparation of this cochlea confirmed earlier that the insertion had been atraumatic: the electrode was visible through the intact basilar membrane and completely located in the scala tympani (Fig. 7C).

In temporal bone 8 , the Contour array pierced the basilar membrane during insertion, and the apical part of the array was positioned in the scala vestibuli. This had been observed during the cochlear surface preparation after insertion (Fig. 8C). On the side view (Fig. 8B) of this temporal bone, the position of the apical electrodes in the scala vestibuli was indicated by the superiorly displaced array in the lower middle and upper basal turn.
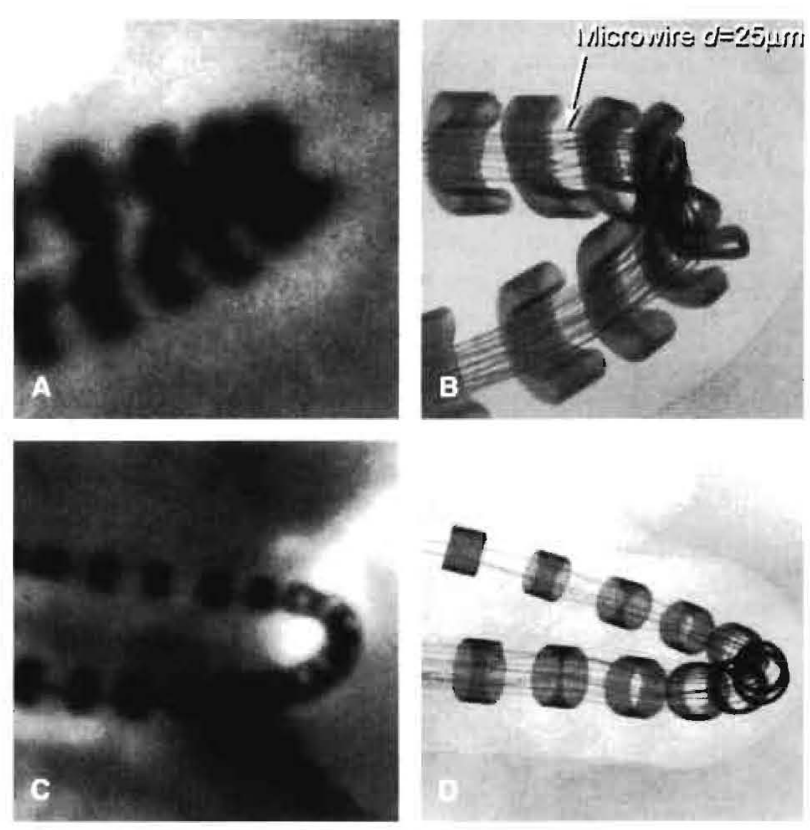

FIG. 4. Enlargements of part of the Nucleus standard electrode array in the cochlea of temporal bone $1(\mathbf{C}, \mathbf{D})$ and the Contour electrode array in the cochlea of temporal bone $4(\mathbf{A}, \mathbf{B})$. Conventional radiographs $(\mathbf{A}, \mathbf{C})$ and phase-contrast radiographs $(\mathbf{B}, \mathbf{D})$. Diameter of microwires, $25 \mu \mathrm{m}$. 

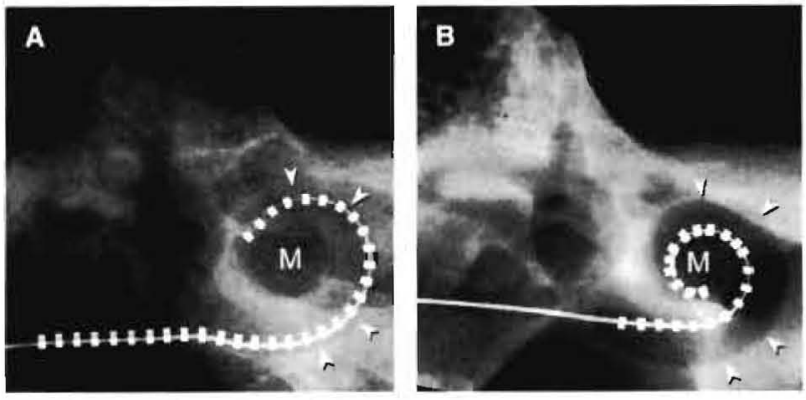

FIG. 5. Phase-contrast radiographs (cochlear view) of temporal bone 1 implanted with the standard array $(\mathbf{A})$ and temporal bone 6 implanted with the Contour array (B) demonstrating that the Contour array was inserted deeper and closer to the modiolus. Arrowhead, outer wall of the cochlea; $M$, modiolus.

\section{DISCUSSION}

In conventional radiography, the yuality of the radiograph is usually improved by reducing the focal spot size and object-image distance. Such an improvement is much more distinct in phase-contrast radiography. which uses a microfocus $x$-ray tube source and large projection distances (Fig. IB). The microfocus source size ensures the sufficiently high level of spatial coherence of the $x$-rays on which the method relies. A very important aspect of this arrangement is the appropriate choice of source-object and object-image distances. Conventional radiography is based on absorption-contrast, whereas phase-contrast radiography is based on phase or refraction effects. Therefore, in conventional radiography, increasing object-image distance will usually degrade the clarity of the image (Fig. 1A). However, large objectimage distances are used to advantage in the phasecontrast technique (Fig. IB).

\section{Improvements of phase-contrast radiography in temporal-bone imaging}

The temporal bone contains a wide range of bone densities and variations in composition. Whereas the highest
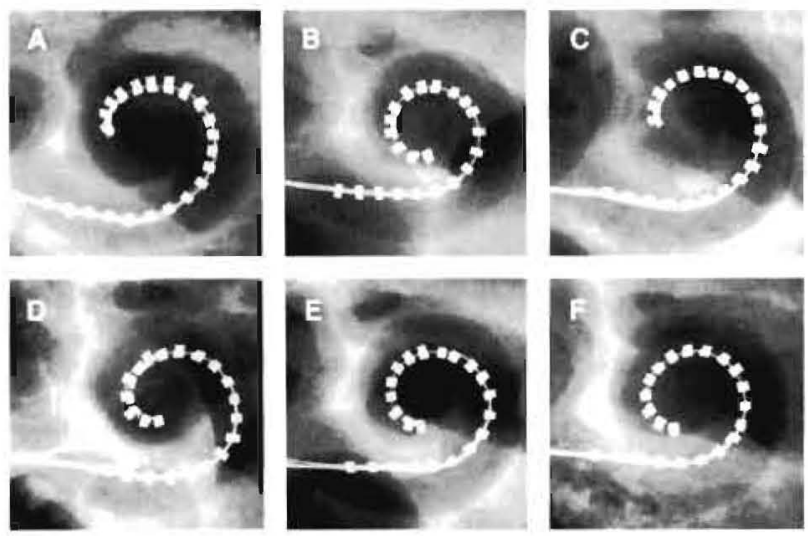

FIG. 6. Phase-contrast radiographs (cochlear view) of cochlear part of temporal bones 5 through 10 showing that the Contour array was generally close to the modiolus.
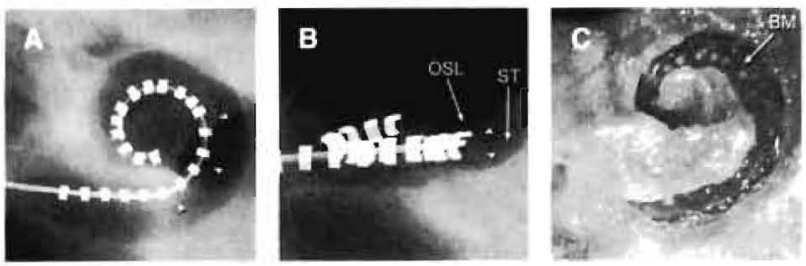

FIG. 7. Example of atraumatic insertion of the Contour array in temporal bone 6 . A. Cochlea view of phase-contrast radiograph. B. Side view of phase-contrast radiograph showing the Contour array located under the osseous spiral lamina (OSL). C. Photomicrograph of the surface prepared cochlea confirming that the electrode array is under the intact basilar membrane (BM). ST, scala tympani; arrowhead, margin of silicone carrier of electrode.

concentration of calcium can be found in the bone surrounding the labyrinth and inside the modiolus, weakly absorbing soft tissue, delicate structures (e.g. membranes. the organ of Corti. osseous spiral lamina) and fluid-filled canals (labyrinth) exist within the temporal bone. Moreover, the temporal bone also has a large range in the geometric dimensions of the various bony components. The maximum dimension of the bony labyrinth, from the posterior end of the lateral semicircular canal to the anterior end of the cochlea, is about $20 \mathrm{~mm}$, whereas the thickness of the OSL is only about $0.1 \mathrm{~mm}$ (unpublished data from our laboratory). Conventional radiography is incapable of showing such fine details of the temporal bone. The results for phase-contrast radiography showed significant improvements in the detail of structures compared with conventional radiography in images of in vitro human temporal bones. These improvements include enhanced contrast at the edge of canal-type leatures, inherent image magnification, higher spatial resolution, improved signal to background (via the so-called air-gap effect) (5) and ability to use detectors such as imaging plates (cf. normal radiographic film without an intensilying screen).

The increase in contrast of the present radiographs of our temporal bone samples could be the result of two mechanisms: I) Edge-enhanced contrast (8) of phasecontrast imaging increases the contrast at the edges of canal-type features. Because the petrous part of the temporal bone is characterized by such canal-type features
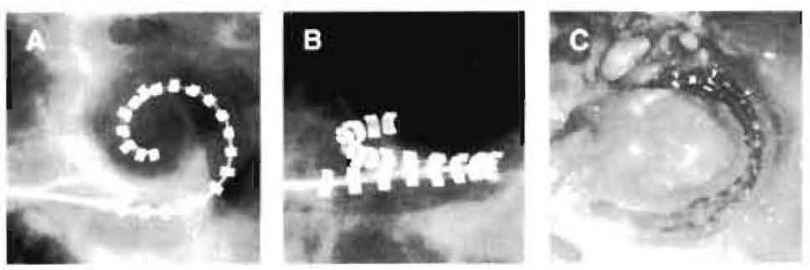

FIG. 8. Example of traumatic insertion of the Contour array in temporal bone 8 . A. Cochlear view of phase-contrast radiograph. B. Side view of phase-contrast radiograph showing the superiorly displaced array in the upper basal turn and the lower middle turn. C. Photomicrograph of the surface prepared cochlea confirming that the Contour array had pierced the basilar membrane (arrowhead). 
(cochlea, vestibule, semicircular canals, facial nerve canal, internal auditory meatus), the anatomic entities, especially in this section of the temporal bone, will be shown more clearly with the new imaging modality. 2) Absorption contrast, which is still present in phasecontrast radiographs, shows best the low spatial frequencies (large features) of an absorptive object and has improved signal to background in the present geometry. The contributions of phase contrast and absorption contrast to the total image contrast are largely complementary, relating to high and low spatial frequencies, respectively, and can therefore provide more information than a conventional radiograph.

In agreement with Wilkins et al. (4) and Gao et al. $(5,8)$, who showed that phase-contrast radiography improves the contrast of weakly absorbing features in small biologic objects, we also noted that phase-contrast radiography improved the contrast of weakly absorbing features within the dense structures of human temporal bone. In particular, delicate structures, such as the OSL, were visible in the side view of temporal bone 6 (Fig. 7B), and nonmetallic materials, such as the silicone carrier of the electrode array, were observed in Figure $7 \mathrm{~A}, \mathrm{~B}$.

The inherent image magnification is another improvement offered by phase-contrast radiography, especially when high spatial resolution is desired. Therefore, phasecontrast radiographs tend to retain sharpness better than do conventional radiographs when enlarged or zoomed (Fig. 4 B,D).

The ability to use imaging plates with higher detective quantum efficiency and a wider dynamic range than conventional radiographic film clearly has advantages of reducing the absorbed dose and increasing the level of information gained. This digital device is ideal for combining with the inherent image magnification of phasecontrast radiography.

\section{Phase-contrast radiography in perimodiolar electrode development}

It has already been demonstrated that the standard Nucleus multichannel electrode array is safe for use in profoundly deaf patients. However, the standard straight array is usually located along the outer wall of the cochlea (Figure 5A). A study by Shepherd et al. (9) has shown that moving the electrode array from the outer wall of the cochlea toward the modiolus, which contains the spiral ganglion cells in the Rosenthal canal, can reduce stimulus thresholds and increase the dynamic range. From a very early stage of our perimodiolar electrode development $(10,11)$, the aim has always been to achieve a perimodiolar placement of the electrode with as little insertion-induced damage as possible (10-12). Cohen et al. (3) developed a method to estimate the position of the electrode within the cochlea using the postoperative radiographs (a cochlear view (1) orientation). The accuracy of this method relies on the visibility of anatomic landmarks on the radiograph. The study described here demonstrates that phase-contrast imaging can have important advantages in the visualization of those anatomic details of both the inner ear structures and the intracochlear electrode array. It therefore can provide a better definition of electrode location in relation to the outer cochlear wall and the modiolus. The method is capable of spatial resolutions on the order of about $1 \mu \mathrm{m}$, because spatial resolution can be comparable to the source size (13). Therefore, phase-contrast radiography is well suited to the investigation of any microelectronic devices.

\section{Clinical application of phase-contrast radiography}

Phase-contrast radiography relies on having high spatial coherence of the x-rays and a very uniform wavefront incident on the feature of interest. The technique is very sensitive to extraneous mechanisms of scattering that either complicates the wavefront or adds background. In practice, this will mean strict quality controls on in-line components such as windows and filters as well as a limit on the thickness of objects to be imaged Nonetheless, previous studies $(4,5)$ have reported experiments demonstrating detectable phase-contrast on softtissue samples $\leq 50 \mathrm{~mm}$ in thickness. Moreover, this study provides support for the feasibility of applying phase-contrast radiography to in vitro human temporal bone. However, its usefulness in large objects or even cochlear implant patients in a clinical setting is still an ongoing computational and physics challenge.

\section{CONCLUSIONS}

This study demonstrates that phase-contrast radiography offers significant improvement over conventional radiography in images of in vitro temporal bones. The phase-contrast imaging provides better visualization of anatomic details of the inner ear and of the structure of the intracochlear electrode array, and better definition of the electrode position in relation to the cochlear wall. It is a potentially valuable tool in the development of new electrode arrays for advanced cochlear implant systems.

Acknowledgments: The authors thank Dr. Yvonne Duan for information, J. Kay for radiographic assistance, Martin Wojak for computing assistance, Cochlear Ltd. for providing the electrodes, X-Ray Technologies Pty., Ltd., for access to their phase-contrast imaging facility, and Fujifilm Co., Ltd., for the use of their BAS-5000 Imaging Plate system.

\section{REFERENCES}

I. Xu J, Xu SA, Cohen LT, et al. Cochlear view: Postoperative radiography for cochlear implantation. Am J Otol 2000;21:49-56.

2. Chen JM, Farb R, Hanusaik L, et al. Depth and quality of electrode insertion: A radiologic and pitch scaling assessment of two cochlear implant systems. Am J Otol 1999;20:192-7.

3. Cohen LT, Xu J, Xu SA, et al. Improved and simplified methods for specifying positions of the electrode bands of a cochlear implant array. Am J Otol 1996;17:859-65.

4. Wilkins SW, Gureyev TE, Gao D, et al. Phase-contrast imaging using polychromatic hard x-rays. Nature 1996;384:335-8.

5. Gao D, Pogany A, Stevenson AW, et al. Phase-contrast radiography. Radiographics 1998;18:1257-67.

6. Xu J, Stevenson AW, Gao D, et al. Phase-contrast radiography: a 
new $\mathrm{x}$-ray technique for cochlear implant research. In: Jahnke $\mathrm{K}$. Fischer M, eds. 4th European Congress of Oto-RhinoLaryngology: Head and Neck Surgery. Vol. 1. Bologna, Italy: Monduzzi Editore, 2000;207-12.

7. Wilkins SW. Simplified conditions and configurations for phase contrast imaging (U.S. Patent No. 08/93(0049). Washington, DC: U.S. Patent and Trademark Office. 1996.

8. Gao D. Pogany A, Stevenson AW. et al. X-ray phase-contras imaging study of sofit tissue and bone samples. In: Boone JM. Dobbins JT, eds. Proceedings of the SPIE: Medical Imaging 1999. Plivsics of Medical limaging. Bellingham. WA: SPIE-The International Society for Optical Engineering. 1999:3659:346-55.
9. Shepherd RK. Hatsushika S, Clark GM. Electrical stimulation of the auditory nerve: The effect of electrode position on neural excitation. Hear Res 1993;66:108-20.

10. Xu SA, Xu J. Seldon HL. et al. Investigation of curved intracochlear electrode arrays. Aust J Otolaryngol 1993;1:276 (abstr).

11. Treaba CG, Xu J, Xu SA. et al. Precurved electrode array and insertion tool. Am Otol Rhinol Laryngol Suppl 1995; I66:438-4I.

12. Tykocinski M, Cohen LT, Pyman BC, et al. Comparison of electrode position in the human cochlea using various perimodiolar electrode arrays. Am J Otol 2000;21:205-11.

13. Pogany A. Gao D. Wilkins $S W$. Contrast and resolution in imaging with a microfocus x-ray source. Rev Sci Instrum 1997:68:2774-82. 


\section{University Library}

\section{- M M N E R VA A gateway to Melbourne's research publications}

Minerva Access is the Institutional Repository of The University of Melbourne

\section{Author/s:}

XU, JIN;Stevenson, Andrew W.;Gao, Dachao;TYKOCINSKI, MICHAEL;LAWRENCE, DAVID;Wilkins, Stephen W.;Clark, Graeme M.;Saunders, Elaine;Cowan, Robert S.

Title:

The role of radiographic phase-contrast imaging in the development of intracochlear electrode arrays

Date:

2001

Citation:

Xu, J., Stevenson, A. W., Gao, D., Tykocinski, M., Lawrence, D., Wilkins, W., et al. (2001). The role of radiographic phase-contrast imaging in the development of intracochlear electrode arrays. Otology and Neurotology, 22, 862-868.

Persistent Link:

http://hdl.handle.net/11343/27592 